\title{
UREM program aims to improve writing and communication
}

\section{mrs.org/diversity}

ate last year, the Materials
Research Society (MRS) announced a new MRS Diversity and Inclusion Fund to support a science-writing program for underrepresented racial and ethnic minority (UREM) students. The goal of the fund is to underwrite a new UREM Science Writing Program in which the students will work with $M R S B u l$ letin staff to develop communication skills and write MRS news articles, blog posts, social media posts, or other communications. Long-time MRS member and volunteer Steven C. Moss provided an initial donation with the hope of rapidly increasing support for the project.

The UREM Science Writing Program is aligned with MRS' Strategic
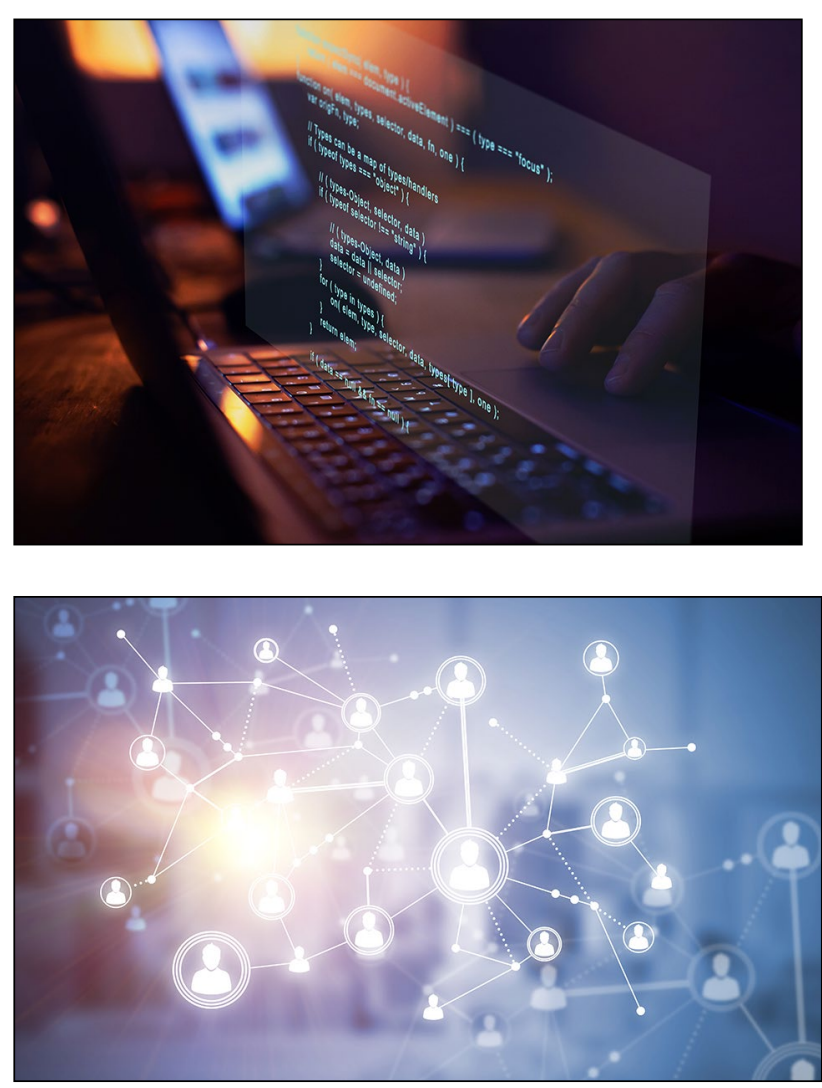

Aspiration to engage members across generations to advance their careers and promote materials research and innovation. The program will provide recipients with student membership in MRS and expose them to both MRS staff and volunteers who can help them grow professionally as materials scientists and remain active, engaged members of the materials community.

Participant Xaimara Santiago Maldonado, University of Puerto Rico, Río Piedras Campus, hopes to better develop skills as a scientific communicator: "I believe the program will allow me to gain valuable experience and knowledge through the development of writing projects in different publishing areas, allowing me to exercise both my analytical and creative thinking. Furthermore, it will help me gain other valuable communication skills in the process as well as networking opportunities that will support my professional growth as a scientist. As a Hispanic scientist, I expect that the skills learned during this program will allow me to further contribute to the spread of scientific knowledge by reaching both English- and Spanish-speaking communities.

"Future participants will benefit from having this immersive writing opportunity in different areas that allow students to be up to date in materials science research, while finding their own creative voice. It could also be beneficial for networking and exploring future professional careers. Moreover, this program gives a voice to underrepresented racial and ethnical minorities so we can keep moving toward a more diverse and equal scientific community," she said.

Many of the students see the program as a way to develop better scientific communication, to articulate their research, and build collaborations with colleagues. "Having other perspectives as scientific writers is important to understand how information is communicated," said Alejandro Burgos-Suazo, PhD student in chemistry at the University of Puerto Rico, Río Piedras Campus.

Social media offers a more fastpaced means to disseminate information. José Arnaldo Méndez Román, teaching assistant at the University of Puerto Rico, Río Piedras Campus, would like to use this medium to inform the public of "important scientific contributions in our society," since it is more accessible to those outside of the science community.

Many of the initial participants attend the same university, showing the value of professors who advocate for professional growth. MRS members are encouraged to share this program and other early-career opportunities as valuable tools in development.

There are opportunities to donate to help in the preparation of these students for future professional and leadership roles in the global materials community. Learn more about the Society's Broadening Participation in Materials Science initiatives and activities that are dedicated to identifying strategies to promote diversity, equity, and inclusion within the broad professional community. 